\title{
Mechanism of action of dicarboximide and phenylpyrrole on the stress-response signal transduction pathway*
}

\author{
Makoto FUJIMURA $^{* *}$ \\ Faculty of Life Sciences, Toyo University, Itakura, Oura, Gunma 374-0193, Japan
}

(Accepted May 21, 2010)

\begin{abstract}
The mechanism of action of dicarboximides and phenylpyrroles has been studied in Neurospora crassa. Both fungicides were found to interfere with the signal transduction pathway composed of the following components: osmotic-sensitive (OS)-1 histidine kinase, histidine phosphotransfer protein (HPT)-1, response regulator protein (RRG)-1, OS-4 mitogen-activated protein kinase (MAPK) kinase kinase, OS-5 MAPK kinase, and OS-2 MAPK. All the components, except HPT-1, were essential for the sensitivity to both fungicides and adaptation to osmotic stress. In contrast, the hpt-1 deletion mutation was lethal unless OS-2 was inactivated. Fludioxonil, osmotic stress, and heat shock induced OS-2 activation by phosphorylation. OS-2 regulated various genes, such as $g c y-1$, which encodes glycerol dehydrogenase; cat-1, which encodes conidial catalase; and the clock-controlled gene (ccg)-1. This implies that the signaling pathway plays an important role not only in the stress response but also in asexual differentiation and circadian output. We found 3 types of dicarboximide-resistant mutations in the $B c O S 1$ gene of Botrytis cinerea, of which the I365S mutation was dominant in the fields. A hybridization probe assay was developed to detect these mutations in a single polymerase chain reaction that may be suitable for monitoring the development of resistance to various fungicides. (C) Pesticide Science Society of Japan
\end{abstract}

Keywords: Neurospora crassa, histidine kinase, MAP kinase, osmotic stress.

\section{Introduction}

Dicarboximide fungicides, such as iprodione and procymidone, have been used to control Botrytis cinerea, a pathogen infecting various vegetables, vines, and flower crops. Dicarboximide-resistant strains of many fungi, such as $B$. cinerea, can be easily isolated under laboratory conditions. Most dicarboximide-resistant laboratory mutants generally acquire high resistance not only to dicarboximides but also to phenylpyrroles, such as fludioxonil, but they are hypersensitive to osmotic stress. In addition, both fungicides induce similar morphological changes in germination tubes, and their fungitoxicity is reduced by piperonyl butoxide and $\alpha$-tocopherol, suggesting that these two types of fungicides have a similar mode of action; however, dicarboximide-resistant strains, isolated from fields, do not show cross-resistance to

\footnotetext{
* See Part II for the full Japanese article.

* To whom correspondence should be addressed.

E-mail:mfujimura@toyonet.toyo.ac.jp

Published online August 7, 2010

(C) Pesticide Science Society of Japan
}

phenylpyrroles or osmotic sensitivity. Despite years of study, the precise mechanism of action of these fungicides is still unclear; however, great progress has been made in elucidation of the molecular basis of signal transduction, which has provided a new insight into the mechanisms of these fungicides. In this article, we review our studies on the mode of action of dicarboximides and phenylpyrroles in Neurospora crassa, and on the molecular mechanisms for field resistance to dicarboximides in B. cinerea.

\section{Fungicide sensitivity based on the stress-response signal transduction pathway in Neurospora}

In $N$. crassa, at least 5 genes, os-1,os-2,os-4,os-5, and cut, are involved in osmotic stress responses. Osmotic stress-sensitive $o s-1, o s-2, o s-4, o s-5$, and cut mutants cannot grow on a medium containing $4 \% \mathrm{NaCl}$. All os mutants were also found to be resistant to iprodione and fludioxonil, whereas cut mutants were as sensitive to the fungicides as wild-type strains. The $o s$ mutants, especially $o s-2, o s-4$, and $o s-5$, had similar phenotypes, such as abnormal morphology of aerial hyphae, stimulated pigmentation on agar slants, and reduced fertility. Both fungicides caused swelling and bursting of the cells and 
glycerol accumulation in the wild-type strain, but not in the os mutants.

Glycerol accumulation in response to hyperosmolarity is an important mechanism for maintaining turgor pressure in the cells. Osmoregulation mediated by the histidine kinase $\operatorname{Sin} 1 \mathrm{p}$ has been well characterized as a high-osmolarity glycerol (HOG) pathway in Saccharomyces cerevisiae. Although budding yeast cells are resistant to iprodione and fludioxonil, a similar signaling pathway in fungi is thought to be the predominant target of fungicides. Studies conducted by our group and others revealed that os genes in $N$. crassa encode components for a pathway similar to the HOG pathway in $S$. cerevisiae. The two-component signal transduction pathway in Neurospora comprises the His-Asp phosphorelay system and the mitogen-activated protein kinase (MAPK) cascade. The phosphorelay system includes OS-1, a sensor histidine kinase; HPT-1 a His-containing phosphotransfer (HPt) protein; and RRG-1, a receiver response regulator. The MAPK cascade includes OS-4 MAPKK kinase, OS-5 MAPK kinase, and OS-2 MAPK. Among them, we identified and characterized $h p t-1, o s-4$, and $o s-5$ genes. The $o s-4$ and $o s-5$ gene mutants had two contrasting phenotypes: hypersensitivity to osmotic stress and resistance to iprodione and fludioxonil; this indicated that proper functioning of this signal transduction pathway is essential for sensitivity to these antifungal compounds.

$N$. crassa has 11 putative histidine kinase genes, including those similar to the yeast $S L N 1$ gene. Among all these genes, mutation in only os-1 confers the properties of fungicide resistance and osmotic sensitivity. $S$. cerevisiae has only one histidine kinase SLN1 gene, and disruption of this kinase is lethal because the Hog1 MAPK pathway is constitutively activated. In contrast, the $o s-1$ gene is not essential for the growth of $N$. crassa under normal hypotonic conditions. OS-1 has 6 unique 90 -amino acid repeats in the sensor region. The $o s-1$ allelic mutants, with mutations in these amino acid repeats, exhibited the pleiotropic phenotypes of fungicide resistance and osmosensitivity. The hpt-1 gene, which encodes only HPt, was successfully disrupted in the $o s-2$ MAPK mutant, but never in the wild-type strain. Genetic analysis revealed that hpt-1 is an essential gene under normal growth conditions and its lethality is suppressed by $o s-2$ or $o s-5$ mutation.

Fludioxonil and iprodione treatments induced the phosphorylation of OS-2 MAPK. In addition, OS-2 was activated in response to multiple stress conditions, including osmotic stress, heat shock, and oxidative stress; however, OS-4 MAPKK kinase and OS-5 MAPK kinase were essential for OS-2 phosphorylation under such multiple stress conditions. In contrast, OS-1 histidine kinase was essential for OS-2 phosphorylation in the presence of fungicides and at low levels of osmotic stress, but this enzyme was not essential under high osmotic and other stresses. The results suggest that these fungicides may exert their antifungal property by overactivating the MAPK cascade downstream of OS-1 histi- dine kinase.

OS-2 MAPK was found to regulate various types of genes that function in the osmotic stress response and cell differentiation. Fludioxonil and osmotic stress induced the synthesis of glycerol by upregulating the expression of $g c y-1$, which encodes a putative glycerol dehydrogenase; this upregulation results in increased enzyme activity of this dehydrogenase. $N$. crassa, like other filamentous fungi, has specific fungal catalases, CAT-1 and CAT-3, predominant in the conidia and mycelia, respectively. Fludioxonil and osmotic stress also induce the expression of cat-1 and its corresponding enzyme activity in the mycelia in an OS-2-dependent manner. In addition, several clock-controlled genes, such as ccg-1, ccg-13, and $b l i-3$, are upregulated by OS-2 activation. These findings imply that the stress-response MAPK cascade plays an important role not only in the osmotic stress response but also in cell differentiation in filamentous fungi.

\section{Identification of the mutations causing resistance to dicarboximides in $B$. cinerea}

In contrast to the os mutants of $N$. crassa, dicarboximide-resistant field isolates of $B$. cinerea did not show osmosensitivity or cross-resistance to phenylpyrroles; however, mutational analysis of $o s-1$ allelic mutants of $N$. crassa revealed that $o s-1$ in these mutants is as highly polymorphic. These results prompted us to clone the os-1-like BcOS1 gene of the dicarboximide-resistant field isolates of $B$. cinerea. We identified a single amino acid substitution, I365S, in BcOS1 histidine kinase, which confers dicarboximide resistance. Dicarboximide-resistant strains that possessed the I365S mutation in $B c O S 1$ were found to be widespread throughout Japan and other countries. We also identified two additional types of dicarboximide-resistant isolates: one with H225P, V368F, Q369H, and T447S, and the other with Q369P and N373S amino acid substitutions in the $\mathrm{BcOS} 1$ gene. These 3 types of dicarboximide-resistant isolates had similar levels of dicarboximide resistance and pathogenicity.

We have developed fluorescence resonance energy transferbased real-time polymerase chain reaction (PCR) assays to detect mutations that confer resistance to dicarboximide and benzimidazole. Our methods are based on the hybridization property and melting curve analysis of DNA. These resistance mutation assays have clear advantages over the commonly used PCR-based procedures, as is evident from the different types of mutations detected: 3 types of dicarboximide-resistant mutations in $\mathrm{BcOS1}$ and 4 types of benzimidazole-resistant mutations in the $\beta$-tubulin gene, in a single PCR reaction without any additional manipulations, such as restriction enzyme digestion and gel electrophoresis after the PCR set-up. Therefore, a PCR-based probe hybridization method would be applicable for the detection of mutations conferring resistance to other fungicides in various pathogens. 


\section{Conclusion}

The mechanisms of action of dicarboximides and resistance to dicarboximides remained unclear even after almost 30 years of research. Our studies indicated that dicarboximides and phenylpyrroles have a unique mode of action; i.e., these fungicides target the stress-response signal transduction pathway. Recently, this pathway has been studied in various filamentous fungi, and these fungicides are being used as a molecular tool to activate this pathway. Further, we identified the histidine kinase $B C O S 1$ mutations that confer dicarboximide resistance in field isolates of $B$. cinerea. Dicarboximide-resistant strains with the I365S mutation in the $\mathrm{BcOS1}$ gene are distributed worldwide. Genotyping of field isolates in Japan suggested that the frequency of dicarboximide-sensitive isolates is increasing because of the decreasing use of dicarboximides as fungicides; this is probably because of the introduction of new fungicides, such as anilinopyrimidines, hydroxyanilides, and QoI fungicides. 